\title{
Efficacy of Compound Kushen Injection in Combination with Induction Chemotherapy for Treating Adult Patients Newly Diagnosed with Acute Leukemia
}

\author{
Honglei Tu, ${ }^{1}$ Bo Lei, ${ }^{2}$ Shan Meng, ${ }^{2}$ Hailing Liu, ${ }^{2}$ Yongchang Wei, ${ }^{3}$ Aili He, \\ Wanggang Zhang, ${ }^{2}$ and Fuling Zhou, ${ }^{1,2}$ \\ ${ }^{1}$ Department of Clinical Hematology, Zhongnan Hospital, Wuhan University, Wuhan, Hubei Province 430071, China \\ ${ }^{2}$ Department of Clinical Hematology, Second Affiliated Hospital, Medical School of Xian Jiaotong University, \\ Xian, Shaanxi Province 710004, China \\ ${ }^{3}$ Department of Radiation and Medical Oncology, Zhongnan Hospital, Wuhan University, Wuhan, Hubei Province 430071, China \\ Correspondence should be addressed to Fuling Zhou; zhoufuling@163.com
}

Received 3 July 2016; Revised 13 August 2016; Accepted 17 August 2016

Academic Editor: Michael Silbermann

Copyright (C) 2016 Honglei Tu et al. This is an open access article distributed under the Creative Commons Attribution License, which permits unrestricted use, distribution, and reproduction in any medium, provided the original work is properly cited.

\begin{abstract}
We assessed the clinical effectiveness and safety of CKI (compound Kushen injection) plus standard induction chemotherapy for treating adult acute leukemia (AL). We randomly assigned 332 patients with newly diagnosed AL to control $(n=165$, receiving DA (daunorubicin and cytarabine) or hyper-CVAD (fractionated cyclophosphamide, doxorubicin, vincristine, and dexamethasone)) or treatment $(n=167$, receiving CKI and DA or hyper-CVAD) groups. Posttreatment, treatment group CD3+, CD4+, CD4+/CD8+, natural killer (NK) cell, and immunoglobulin (IgG, IgA, and IgM) levels were significantly higher than those of the control group $(p<0.05)$, and CD8+ levels were lower in the treatment group than in the control group $(p<0.05)$. Treatment group interleukin(IL-) 4 and IL-10 levels were significantly higher compared to the control posttreatment (both $p<0.05$ ) as were complete remission, overall response, and quality of life (QoL) improvement rates $(p<0.05)$. The control group had more incidences of grade $3 / 4$ hematologic and nonhematologic toxicity $(p<0.05)$. Responses to induction chemotherapy, QoL improvement, and adverse events incidence between control group patients with acute myeloid leukemia and acute lymphocytic leukemia were not significantly different. CKI plus standard induction chemotherapy is effective and safe for treating AL, possibly by increasing immunologic function.
\end{abstract}

\section{Introduction}

Acute leukemia (AL), a malignant neoplasm, is characterized by clonal blood cell proliferation within the bone marrow. Historically, AL diagnosis is linked with poor prognosis, particularly in older adults. Improved disease treatment and management have led to increased overall survival trends [1]. The Surveillance Epidemiology and End Results reported $24 \%$ and $65 \%$ 2002-2008 relative five-year survival rates in adults with acute myeloid leukemia (AML) or acute lymphoblastic leukemia (ALL), respectively [2]. The nature of adult AL necessitates emergent, aggressive inpatient chemotherapy. However, standard chemotherapy is not always tolerated by patients; the adverse events during chemotherapy, such as myelosuppression, gastrointestinal reaction, infection, and cardiotoxicity, often lead to interruption of chemotherapy [3]; consequently, there is treatment failure. Therefore, such patients urgently need effective, lowtoxicity therapy.

Therapy integrating traditional Chinese and Western medicine has been the most distinctive method for treating malignant tumors in China [4-7]. As a representative of traditional Chinese medicine (TCM), compound Kushen injection (CKI) is extracted from the Kushen (Radix Sophorae Flavescentis) and Baituling (Rhizoma smilacis Glabrae) herbs; its primary components are oxymatrine and matrine $[8,9]$. Additional File 1 (in Supplementary Material available online at http://dx.doi.org/10.1155/2016/3121402) contains the CKI 
fingerprint. The State Food and Drug Administration of China approved CKI for treating cancer in 1992 [10]. Since then, CKI has been extensively used in the Chinese clinical setting. There are many clinical reports demonstrating its anticancer effect and these reports include using CKI to treat gastric cancer, liver cancer, lung cancer, breast cancer, ovarian cancer, colorectal cancer, and other cancer types [1114]. It is reported that CKI attenuates chemotherapy and radiotherapy side effects by improving quality of life (QoL), regulating immune function, and synergizing chemotherapy and radiotherapy therapeutic effects $[15,16]$. In addition, it is reported that there is a positive effect of CKI on bone cancer pain: compared with radiotherapy or bisphosphonates, CKI showed significant effects on the improvement of pain relief in patients with bone cancer pain and the increase in Karnofsky Performance Status (KPS). The patients treated with CKI achieved statistically significant reductions in the incidence of leukopenia and nausea. No severe adverse events were found and no treatment was stopped because of adverse events of CKI in the treatment groups [17]. However, the scientific literature contains scarce empirical data involving CKI in ALs, and the underlying complex mechanisms of its anticancer effect are not fully understood. In the study, our primary aim was to determine whether CKI with induction chemotherapy has a similar or better effect in improving the objective response rate (ORR) in adult AL. The secondary aims were to compare immunologic function, quality of life (QoL), clinical symptoms, and adverse drug reactions (ADRs) and to explore the reasons therein.

\section{Materials and Methods}

2.1. Study Groups. We selected 332 previously untreated patients (aged 18-78 years) with AL who were treated at our hospital between January 2011 and January 2016. According to the World Health Organization criteria, 175 and 157 patients were diagnosed with AML and ALL, respectively. The patients were randomly assigned to a treatment $(n=$ 167 ) or control ( $n=165)$ group. No patient had central nervous system diseases, other malignancies, or uncontrolled infections. They also had no inflammatory conditions, pathological antecedents, or endocrine dysfunctions. We excluded patients with known confusion or who were deemed too ill to participate. The Wuhan University Ethics Committee approved this study and it met international standards for patient confidentiality. All patients signed informed consent forms according to the Declaration of Helsinki.

2.2. Treatment Methods. Both groups received induction chemotherapy, in which patients with confirmed AML received the DA regimen, and patients with ALL received the hyper-CVAD regimen. The treatment group received CKI in addition to the DA or hyper-CVAD regimens.

The DA regimen consisted of $60 \mathrm{mg} / \mathrm{m}^{2}$ daunorubicin on days $1-3$ and $200 \mathrm{mg} / \mathrm{m}^{2}$ cytarabine on days $1-7$. Hyper-CVAD treatment consisted of $300 \mathrm{mg} / \mathrm{m}^{2}$ fractionated cyclophosphamide twice daily on days $1-3,50 \mathrm{~g} / \mathrm{m}^{2}$ doxorubicin on day 4, $2 \mathrm{mg}$ vincristine on days 4 and 11, and $40 \mathrm{mg}$ dexamethasone on days 1-4 and 11-14. In the treatment group, according to the instructions, $20 \mathrm{~mL}$ CKI (Shanxi Zhendong Pharmacy Limited Company, Chinese medicine permit number Z14021231) plus $200 \mathrm{~mL}$ saline was administered by intravenous drip, at 40-60 drips each min, once daily for 14 days. After the end of the first induction course, a bone marrow biopsy was used to assess the treatment response. Patients with complete remission (CR) received continued consolidation therapy or autologous or allogeneic stem cell transplantation, and patients who did not achieve CR in 2 cycles had their induction programs adjusted.

2.3. Observation Indices. The present analysis involved only 12 cycle of induction chemotherapy. Treatment response, QoL, and toxicity (hematologic and nonhematologic (including gastrointestinal reaction, pneumonia, hepatotoxicity, neurological dysfunction, renal dysfunction, and skin reactions)) were evaluated. One day before treatment and 1 week after the end of the treatment course in both groups, the peripheral blood T-lymphocyte subgroup (CD3+, CD4+, and $\mathrm{CD} 8+$ ), natural killer (NK) cell, and immunoglobulin (IgG, IgA, and IgM) levels were detected with flow cytometry, the CD4+/CD8+ ratios were calculated, and cytokines (interleukin- (IL-) 4 and IL-10) were detected by enzymelinked immunosorbent assay (ELISA).

2.4. Statistical Analysis. We used SPSS version 20.0 for the statistical analyses. All data are presented as the mean \pm standard deviation $(x \pm s)$. The groups were compared using independent sample $t$-tests. The differences in characteristics between the two groups were examined using the $\chi^{2}$ test. $p<$ 0.05 was considered significant.

\section{Results}

3.1. Baseline Characteristics. The clinicopathological information of the treatment and control groups did not significantly differ $(p>0.05$, Table 1$)$.

3.2. Changes in Immune Function Indices. The peripheral blood CD3+, CD4+, CD8+, CD4+/CD8+, NK cell, and immunoglobulin levels in the treatment and control groups did not differ significantly 1 day before treatment $(p>$ 0.05). After the first course of induction chemotherapy, in the control group, CD3+, CD4+, CD4+/CD8+, NK cell, and immunoglobulin (IgA, IgM, and $\operatorname{IgG}$ ) levels decreased significantly $(p<0.05)$ and CD8+ was increased significantly $(p<0.05)$; in the treatment group, levels of CD3+, IgA, and IgM decreased significantly $(p<0.05), \mathrm{CD} 4+$ was increased significantly $(p<0.05)$, and CD8+, CD4+/CD8+, NK cell, and IgG levels did not change significantly $(p>0.05)$. This suggests that immunity function has been decreased in AL patients with induction chemotherapy. The serum CD3+, $\mathrm{CD} 4+, \mathrm{CD} 4+/ \mathrm{CD} 8+$, NK cells, and immunoglobulin levels were significantly lower in the control group than in the treatment group $(p<0.05$, Table 2$)$, which implies that CKI improves immunologic function in patients with AL. 
TABLE 1: Comparison of main status between treatment and control groups $(n)$.

\begin{tabular}{|c|c|c|c|c|}
\hline Variable & $\begin{array}{l}\text { Treatment } \\
\text { group } \\
(n=167)\end{array}$ & $\begin{array}{l}\text { Control } \\
\text { group } \\
(n=165)\end{array}$ & $\chi^{2}$ & $p$ \\
\hline \multicolumn{5}{|l|}{ Age (years) } \\
\hline$\leq 60$ & 78 & 74 & \multirow{2}{*}{0.115} & \multirow{2}{*}{0.734} \\
\hline$>60$ & 89 & 91 & & \\
\hline \multicolumn{5}{|l|}{ Sex } \\
\hline Men & 87 & 88 & \multirow{2}{*}{0.015} & \multirow{2}{*}{0.821} \\
\hline Women & 80 & 77 & & \\
\hline \multicolumn{5}{|l|}{ AML } \\
\hline M1 & 3 & 4 & \multirow{5}{*}{0.306} & \multirow{5}{*}{0.989} \\
\hline M2 & 11 & 10 & & \\
\hline M4 & 39 & 40 & & \\
\hline M5 & 29 & 28 & & \\
\hline M6 & 6 & 5 & & \\
\hline \multicolumn{5}{|l|}{ ALL } \\
\hline L1 & 44 & 44 & \multirow{3}{*}{0.013} & \multirow{3}{*}{0.993} \\
\hline $\mathrm{L} 2$ & 26 & 25 & & \\
\hline L3 & 9 & 9 & & \\
\hline \multicolumn{5}{|l|}{ KPS } \\
\hline$\geq 90$ & 17 & 21 & \multirow{3}{*}{1.299} & \multirow{3}{*}{0.522} \\
\hline $70-90$ & 116 & 105 & & \\
\hline$\leq 70$ & 34 & 39 & & \\
\hline
\end{tabular}

Notes: AML: acute myeloid leukemia, ALL: acute lymphocytic leukemia, and KPS: Karnofsky Performance Status.

3.3. Cytokine Changes. Before treatment, the treatment and control groups did not significantly differ in the peripheral blood counts for IL-4 and IL-10 ( $p>0.05)$. After the end of treatment, IL-4 and IL-10 levels were increased significantly in the treatment group and were significantly higher than those of the control group (both $p<0.05$ ); however, those of the control group were decreased significantly compared to pretreatment levels $(p<0.05$, Table 2$)$.

3.4. Response to Induction Chemotherapy. Our evaluation was based on the National Comprehensive Cancer Network (NCCN) Guidelines (Version 1. 2014) for evaluating response criteria for blood and bone marrow in AL: CR (no circulating blasts or extramedullary disease, trilineage hematopoiesis $(\mathrm{TLH})<5 \%$ blasts, absolute neutrophil count (ANC) > $1000 / \mu \mathrm{L}$, platelets $>1000,000 / \mu \mathrm{L}$, and no recurrence for 4 weeks); CRi (CR with incomplete blood count recovery: recovery of platelets but $<1000,000$ or $\mathrm{ANC}=1000 / \mu \mathrm{L}$ ); ORR $(\mathrm{ORR}=\mathrm{CR}+\mathrm{CRi})$. We assessed disease status via bone marrow biopsies performed 14 days ( \pm 2 days) after induction chemotherapy. After the first cycle of induction chemotherapy, the treatment group ORR rate was significantly higher than that of the control (AML: $92 \%$ versus $73.6 \%, p<0.05$; ALL: $89.9 \%$ versus $69.2 \%, p<0.05$ ) (Table 3 ), which showed that CKI plus chemotherapy significantly improved the ORR as compared with induction chemotherapy alone.
3.5. Evaluation of QoL. QoL evaluation was according to the Karnofsky Performance Status (KPS) and was classified as improvement (improved KPS of $\geq 10$ points posttreatment); stabilization (improved or decreased KPS of $<10$ points posttreatment); deterioration (decreased KPS of $\geq 10$ points posttreatment). Before treatment, the two groups patients did not significantly differ in KPS $(p>0.05$, Table 1$)$. After completing the first cycle of induction therapy, the treatment group KPS improvement rate was significantly higher than that of the control (AML: $55.7 \%$ versus $28.7 \%, p<0.05$; ALL: $59.5 \%$ versus $24.4 \%, p<0.05$ ), and KPS deterioration rate was significantly lower in the treatment group than in the control (AML: $9.1 \%$ versus $28.7 \%, p<0.05$; ALL: $11.4 \%$ versus $38.5 \%$, $p<0.05$ ) (Table 4), indicating that CKI plus chemotherapy can improve patient QoL.

3.6. Toxicity Reactions. According to the National Cancer Institute (NCI) Common Terminology Criteria for Adverse Events (CTCAE) v 3.0 [18], drug toxicity and adverse reactions were classified into four grades (1-4). Grade 1/2 adverse reactions were observed in many patients and were not considered in this study.

Whether in patients with AML or ALL, there was more hematologic toxicity in the control group $(p<0.05)$. Thrombocytopenia and anemia were observed in 9 patients $(5.4 \%)$ in the treatment group as compared with 38 patients (23\%) in the control group, and the patients received blood platelet or red blood cell transfusions $(p<0.05)$. Similarly, nonhematologic toxicity occurred significantly more often in the control group. In total, 2 patients $(1.2 \%)$ in the treatment group compared with 15 patients $(9.1 \%)$ in the control group developed grades 3 and 4 pneumonia $(p<0.05)$; 5 control group patients died of pneumonia during treatment; no treatment group patient died. The incidences of adverse events between patients with AML and ALL in the same treatment groups were not significantly different (Table 5).

The results show that chemotherapy-related complications are reduced following CKI plus chemotherapy as compared with chemotherapy alone.

\section{Discussion}

Traditional Chinese Medicine (TCM) holds that, in treating tumors, eliminating pathogenic factors and strengthening genuine Qi are of equal importance: eliminating pathogenic factors is killing tumor cells by using radiotherapy or chemotherapy and strengthening genuine Qi protects immunologic functions of the organism by using drugs, increasing immunity of the organism $[19,20]$. Hematopoietic stem cells that have lost the normal differentiation capacity into mature blood cells give rise to leukemia [21, 22]. In AL treatment, induction chemotherapy to achieve CR is considered basic therapy [23]. However, chemotherapy cannot avoid injuring the immune system, which mainly manifests in the fact that chemotherapy drugs directly or indirectly kill the immunologic effector cells, leading to decreased immune function $[24,25]$. Antitumor immune responses are T-cellmediated specific responses. T-cells can be divided into CD4+ 
TABLE 2: Comparison of immune function indices and cytokines in the treatment and control groups before and after treatment $(x \pm s)$.

\begin{tabular}{lcccc}
\hline \multirow{2}{*}{ Variable } & \multicolumn{2}{c}{ Treatment group $(n=167)$} & \multicolumn{2}{c}{ Control group $(n=165)$} \\
& Before treatment & After treatment & Before treatment & $34.29 \pm 4.93^{\mathrm{b}}$ \\
\hline CD3+ & $56.43 \pm 4.22$ & $55.45 \pm 4.22^{* \mathrm{a}}$ & $55.38 \pm 5.33$ & $17.13 \pm 5.81^{\mathrm{b}}$ \\
CD4+ & $31.55 \pm 5.33$ & $35.35 \pm 4.24^{* \mathrm{a}}$ & $33.19 \pm 5.39$ & $15.23 \pm 3.03^{\mathrm{b}}$ \\
CD8+ & $13.32 \pm 2.33$ & $13.12 \pm 2.23^{*}$ & $14.31 \pm 3.02$ & $1.08 \pm 0.33^{\mathrm{b}}$ \\
CD4+/CD8+ & $1.69 \pm 0.33$ & $1.73 \pm 0.22^{*}$ & $15.12 \pm 3.72$ & $10.12 \pm 3.11^{\mathrm{b}}$ \\
NK cells & $15.44 \pm 3.35$ & $15.41 \pm 4.02^{*}$ & $12.4 \pm 1.3$ & $7.2 \pm 1.5^{\mathrm{b}}$ \\
IgG & $12.5 \pm 1.3$ & $12.3 \pm 1.2^{*}$ & $2.4 \pm 0.3$ & $1.2 \pm 0.5^{\mathrm{b}}$ \\
IgA & $2.4 \pm 0.3$ & $2.3 \pm 0.3^{* \mathrm{a}}$ & $1.81 \pm 0.47$ & $0.45 \pm 0.53^{\mathrm{b}}$ \\
IgM & $1.71 \pm 0.30$ & $1.58 \pm 0.4^{* \mathrm{a}}$ & $22.5 \pm 3.2$ & $11.8 \pm 2.2^{\mathrm{b}}$ \\
IL-4 & $20.0 \pm 2.4$ & $22.2 \pm 2.3^{* \mathrm{a}}$ & $18.90 \pm 2.1$ & $9.80 \pm 3.2^{\mathrm{b}}$ \\
IL-10 & $17.10 \pm 2.2$ & $19.4 \pm 4.2^{* \mathrm{a}}$ & \\
\hline
\end{tabular}

Notes: compared with the control group after treatment, ${ }^{*} p<0.05$; compared with the treatment group before treatment, ${ }^{\mathrm{a}} p<0.05$; compared with the control group before treatment, ${ }^{\mathrm{b}} \mathrm{p}<0.05$.

TABLE 3: Response to induction chemotherapy in the treatment and control groups.

\begin{tabular}{lcccc}
\hline \multirow{2}{*}{ Variable } & \multicolumn{2}{c}{ AML (\%) } & \multicolumn{2}{c}{ ALL (\%) } \\
& Treatment group $(n=88)$ & Control group $(n=87)$ & Treatment group $(n=79)$ & Control group $(n=78)$ \\
\hline CR (\%) & $72(81.8)$ & $58(66.7)$ & $64(81)$ & $51(65.4)$ \\
CRi (\%) & $9(10.2)$ & $6(6.9)$ & $7(8.9)$ & $3(3.8)$ \\
ORR (\%) & $81(92)^{\mathrm{a}}$ & $64(73.6)$ & $71(89.9)^{\mathrm{a}}$ & $54(69.2)$ \\
\hline
\end{tabular}

Notes: AML: acute myeloid leukemia, ALL: acute lymphocytic leukemia, CR: complete remissions, CRi: CR with incomplete blood count recovery, and ORR: overall response rate. Compared with the control group, ${ }^{\mathrm{a}} p<0.05$.

TABLE 4: KPS index changes of two groups before and after treatment.

\begin{tabular}{lcccc}
\hline \multirow{2}{*}{ Variable } & \multicolumn{2}{c}{ AML (\%) } & \multicolumn{2}{c}{ ALL (\%) } \\
& Treatment group $(n=88)$ & Control group $(n=87)$ & Treatment group $(n=79)$ & Control group $(n=78)$ \\
\hline Improvement (\%) & $49(55.7)^{\mathrm{a}}$ & $25(28.7)$ & $47(59.5)^{\mathrm{a}}$ & $19(24.4)$ \\
Stabilization (\%) & $31(35.2)$ & $37(42.5)$ & $23(29.1)$ & $29(37.2)$ \\
Deterioration (\%) & $8(9.1)^{\mathrm{a}}$ & $25(28.7)$ & $9(11.4)^{\mathrm{a}}$ & $30(38.5)$ \\
\hline
\end{tabular}

Notes: AML: acute myeloid leukemia, ALL: acute lymphocytic leukemia. Compared with the control group, ${ }^{\mathrm{a}} p<0.05$.

TABLE 5: Grade 3/4 drug toxicity and adverse events occurring during the induction period in the treatment and control groups.

\begin{tabular}{lcccc}
\hline \multirow{2}{*}{ Variable } & \multicolumn{2}{c}{ AML (\%) } & \multicolumn{2}{c}{ ALL (\%) } \\
& Treatment group $(n=88)$ & Control group $(n=87)$ & Treatment group $(n=79)$ & Control group $(n=78)$ \\
\hline Hematologic toxicity & $6(6.8)^{\mathrm{a}}$ & $21(24.1)$ & $3(3.8)^{\mathrm{a}}$ & $17(21.8)$ \\
Gastrointestinal reaction & $2(2.3)^{\mathrm{a}}$ & $10(11.5)$ & $1(1.3)^{\mathrm{a}}$ & $8(10.3)$ \\
Pneumonia & $1(1.1)^{\mathrm{a}}$ & $9(10.3)$ & $1(1.3)$ & $6(7.7)$ \\
Cardiotoxicity & $0(0)$ & $4(4.6)$ & $1(1.3)$ & $5(6.4)$ \\
Hepatotoxicity & $3(3.4)^{\mathrm{a}}$ & $11(12.6)$ & $2(2.5)^{\mathrm{a}}$ & $9(11.5)$ \\
Neurological dysfunction & $1(1.1)^{\mathrm{a}}$ & $7(8)$ & $1(1.3)^{\mathrm{a}}$ & $8(10.3)$ \\
Renal dysfunction & $3(3.4)^{\mathrm{a}}$ & $16(18.4)$ & $1(1.3)^{\mathrm{a}}$ & $11(14.1)$ \\
Skin reactions & $1(1.1)^{\mathrm{a}}$ & $7(8)$ & $0(0)^{\mathrm{a}}$ & $5(6.4)$ \\
\hline
\end{tabular}

Notes: AML: acute myeloid leukemia, ALL: acute lymphocytic leukemia. Compared with the control group, ${ }^{\mathrm{a}} p<0.05$.

helper/induced T-cells and CD8+ suppressor/killer T-cells at a $\mathrm{CD} 4+/ \mathrm{CD} 8+$ ratio of $1.5-2.0$. When the immunologic function in patients with cancer is impaired, CD4+ cells will decrease, CD8+ cells will increase, and the CD4+/CD8+ ratio decreases or even inverts [26]. CD3+ cells reflect the total CD4 and CD8 levels. When cellular immunologic function decreases, the NK cells ratio will also decrease, as they are unable to effectively kill tumor cells. Besides, immunoglobulins (Ig) also play an important role in adaptive immunity [27]. Therefore, flow cytometry determination of 
the changes in peripheral blood T-cell subgroups, NK cells, and immunoglobulins (IgG, IgA, and IgM) can illustrate the changes in immunologic function.

In our study, T-cell subgroups, NK cells, and immunoglobulins decreased after induction chemotherapy in the control group as compared with pretreatment levels, indicating that chemotherapy has an inhibitory action on immunologic function; however, among patients in the treatment group, the T-cell subgroups, NK cells, and immunoglobulin levels were significantly higher than those of the control group, suggesting that CKI protects the immune functions of patients who undergo chemotherapy, which is similar to previous research results. In addition, after induction chemotherapy, the treatment group QoL improvement rate was higher than that in the control group. Therefore, we conclude that CKI aids antitumor therapy, resists the toxicity of chemotherapy drugs, and improves the QoL by increasing the immune system function in patients with $\mathrm{AL}$ who receive chemotherapy. Also, our results showed that the treatment group CR and ORR were higher than those in the control after 1-2 cycles of induction chemotherapy, whereas the longterm effect regarding treatment response and survival needs further investigation. During induction chemotherapy, no any special adverse events were observed and no treatment was stopped because of adverse events of CKI in the treatment group, and the incidence of grades 3 and 4 chemotherapyrelated toxicity was quite low in the treatment group than in the control, which means that CKI is safe for patients with $\mathrm{AL}$ and its primary active components oxymatrine and matrine have no direct interactions with used chemotherapies prescription drugs.

Traditional Chinese Medicine (TCM) is mostly prescribed in combination to obtain synergistic effects and reduce possible adverse reactions $[28,29]$. Hence, the compatibility of Chinese medicinal herbs is an important theory in the combination of TCM. According to the record of Chinese herbal medicine toxicology database, Sophorae flavescentis root and seeds are poisonous; the symptom of poisoning is given priority with the nervous system, which performed for salivation, breathing and pulse acceleration, gait instability, serious eclampsia, or death from respiratory depression [30]. So reducing the toxicity of Sophorae flavescentis radix is helpful to improve the safety of clinical medication. Glycyrrhizae radix is prescribed in many Chinese traditional formulas for its medical potential effects in antiinflammation [31], immunoregulation [32], and antiallergy nature [33]. Long-term clinical practice has also confirmed that Glycyrrhizae radix has certain detoxification effect for a variety of poisoning from drugs, animals, or the body's metabolic products [34]. In addition, after compatibility of Sophorae flavescent radix and Glycyrrhizae radix, the mortality of mice was reduced, and there were changes in the content of four kinds of indicator elements, brain tissue, and liver tissue pathology $[35,36]$. Future studies may combine them together to determine the interaction of Sophorae flavescentis radix and Glycyrrhizae radix.

We acknowledge that there are some limitations to the current study. First, patients of all ages were enrolled, which resulted in a heterogeneous distribution of adjuvant treatment procedures. Additionally, the duration of followup was too short for CKI. We adjusted the treatment programs for patients who did not achieve CR within 2 cycles, and patients with CR received consolidation therapy or autologous or allogeneic stem cell transplantation and were no longer evaluated in the study. We may state that induction chemotherapy is only one important part of leukemia treatment, and follow-up treatment is directly related to survival, for which there may also be many uncertain factors closely based on mutations related to the patients themselves.

\section{Conclusions}

In summary, CKI combined with proven induction chemotherapy regimens for AL appears to have better short-term efficacy and lower toxicity, which may depend on increasing the patient's immunologic function and improving the QoL. In Chinese clinics, we commonly administer CKI to synergize the therapeutic effects of chemotherapy or radiotherapy. Whether CKI can prolong the survival of patients with AL requires further investigation.

\section{Competing Interests}

The authors declare that there is no conflict of interests regarding the publication of this paper.

\section{Acknowledgments}

The authors thank all the patients who participated in this study.

\section{References}

[1] D. Pulte, A. Gondos, and H. Brenner, "Expected long-term survival of patients diagnosed with acute myeloblastic leukemia during 2006-2010," Annals of Oncology, vol. 21, no. 2, pp. 335341,2010 .

[2] N. Howlader, A. M. Noone, M. Krapcho et al., SEER Cancer Statistics Review, 1975-2009, National Cancer Institute, Bethesda, Md, USA, 2012.

[3] J. M. Vagace and G. Gervasini, "Chemotherapy toxicity in patients with acute leukemia," in Acute Leukemia-The Scientist's Perspective and Challenge, M. Antica, Ed., chapter 17, InTech, Rijeka, Croatia, 2011.

[4] "Challenges in research and development of traditional chinese medicines," Chinese Herbal Medicines, no. 1, pp. 1-28, 2009.

[5] Q. Ji, Y. Luo, W. Wang, X. Liu, Q. Li, and S. Su, "Research advances in traditional Chinese medicine syndromes in cancer patients," Journal of Integrative Medicine, vol. 14, no. 1, pp. 12-21, 2016.

[6] C.-L. Qian, F. Yan, Y.-Z. Song, D. Li, K.-Z. Dong, and Y.-M. Zhu, "Is the traditional Chinese medicine helpful for patients with hematologic malignant diseases? A meta-analysis of randomized controlled trials," World Journal of Meta-Analysis, vol. 3, no. 3, pp. 163-180, 2015. 
[7] Z. Liu, S. Chen, J. Cai et al., "Traditional Chinese Medicine syndrome-related herbal prescriptions in treatment of malignant tumors," Journal of Traditional Chinese Medicine, vol. 33, no. 1, pp. 19-26, 2013.

[8] J. Tian, W.-H. Wang, H.-M. Cao, and Z.-M. Wang, "Determination of matrine, sophoridine and oxymatrine in compound Kushen injection by HPLC," China Journal of Chinese Materia Medica, vol. 32, no. 3, pp. 222-224, 2007.

[9] Q. I. Liang, J. Zhang, and Z. Zhang, "Determination of four alkaloids in compound Kushen Injection by high performance liquid chromatography with ionic liquid as mobile phase additive," Chinese Journal of Chromatography, vol. 31, no. 3, pp. 249-253, 2013.

[10] Z. Z. Qian, Y. Dan, Y. Z. Liu, and Y. Peng, "Pharmacopoeia of the People's Republic of China (2010 edition): a milestone in development of China's healthcare," Chinese Herbal Medicines, vol. 2, no. 2, pp. 157-160, 2010.

[11] M. Sun, H. Cao, L. Sun et al., "Antitumor activities of kushen: literature review," Evidence-Based Complementary and Alternative Medicine, vol. 2012, Article ID 373219, 11 pages, 2012.

[12] X. Ma, R.-S. Li, J. Wang et al., "The therapeutic efficacy and safety of compound kushen injection combined with transarterial chemoembolization in unresectable hepatocellular carcinoma: an update systematic review and meta-analysis," Frontiers in Pharmacology, vol. 7, article 70, 2016.

[13] W. Xu, H. Lin, Y. Zhang et al., "Compound Kushen Injection suppresses human breast cancer stem-like cells by downregulating the canonical Wnt/ $\beta$-catenin pathway," Journal of Experimental and Clinical Cancer Research, vol. 30, no. 1, article 103, 2011.

[14] L. Yin, W. L. Zheng, Q. Z. Sun, Y. F. Peng, and M. A. Wen$\mathrm{Li}$, "Meta-analysis of efficiency in treatment of gastric cancer by compound Kushen injection combined with chemotherapy," Chinese Journal of Experimental Traditional Medical Formulae, vol. 19, no. 14, pp. 325-330, 2013.

[15] Z. Y. Wang, G. S. Li, and H. X. Huang, "Clinical observation on treatment of 75 mid-late stage cancer patients with Yanshu injection," Chinese Journal of Integrated Traditional \& Western Medicine, vol. 26, no. 8, pp. 681-684, 2006.

[16] J. Chen, Q. Mei, Y.-C. Xu, J. Du, Y. Wei, and Z.-M. Xu, "Effects of Matrine injection on T-lymphocyte subsets of patients with malignant tumor after gamma knife radiosurgery," Journal of Chinese Integrative Medicine, vol. 4, no. 1, pp. 78-79, 2006.

[17] B. Yanju, L. Yang, B. Hua et al., "A systematic review and metaanalysis on the use of traditional Chinese medicine compound kushen injection for bone cancer pain," Supportive Care in Cancer, vol. 22, no. 3, pp. 825-836, 2014.

[18] L. Guerretta, "Common Terminology Criteria for Adverse Events v3.0 (CTCAE)," 2003.

[19] R. Guangxin, L. I. Hegen, L. Zhao, L. Liu, L. Shen, and L. Zhou, "Effect of method of strengthening genuine qi on tumor microenvironment of advanced non-small cell lung cancer," Journal of Liaoning University of Traditional Chinese Medicine, no. 6, pp. 157-159, 2013.

[20] M. Xu, G. Zhang, L. Pan et al., "Effects of Chinese herbs of strengthening and invigorating genuine Qi on multidrug resistant lung cancer cells through regulation of mitochondria related molecules," Chinese Journal of Clinical Oncology, vol. 37, no. 13, pp. 725-728, 2011.

[21] M. R. Corces-Zimmerman and R. Majeti, "Pre-leukemic evolution of hematopoietic stem cells: the importance of early mutations in leukemogenesis," Leukemia, vol. 28, no. 12, pp. 2276-2282, 2014.

[22] S. Kanji, V. J. Pompili, and H. Das, "Plasticity and maintenance of hematopoietic stem cells during development," Recent Patents on Biotechnology, vol. 5, no. 1, pp. 40-53, 2011.

[23] A. Nazha and F. Ravandi, "Acute myeloid leukemia in the elderly: do we know who should be treated and how?" Leukemia \& Lymphoma, vol. 55, no. 5, pp. 979-987, 2014.

[24] C. Rébé and F. Ghiringhelli, "Cytotoxic effects of chemotherapy on cancer and immune cells: how can it be modulated to generate novel therapeutic strategies?" Future Oncology, vol. 11, no. 19, pp. 2645-2654, 2015.

[25] R. G. van der Most, A. Currie, B. W. S. Robinson, and R. A. Lake, "Cranking the immunologic engine with chemotherapy: using context to drive tumor antigen cross-presentation towards useful antitumor immunity," Cancer Research, vol. 66, no. 2, pp. 601-604, 2006.

[26] P. Olioso, R. Giancola, M. Di Riti, A. Contento, P. Accorsi, and A. Iacone, "Immunotherapy with cytokine induced killer cells in solid and hematopoietic tumours: a pilot clinical trial," Hematological Oncology, vol. 27, no. 3, pp. 130-139, 2009.

[27] S. Alanko, T.-T. Pelliniemi, and T. T. Salmi, "Recovery of blood B-lymphocytes and serum immunoglobulins after chemotherapy for childhood acute lymphoblastic leukemia," Cancer, vol. 69, no. 6, pp. 1481-1486, 1992.

[28] X. Liu, H. Li, L. Wu et al., "Simultaneous quantification of chrysophanol and physcion in rat plasma by ultra fast liquid chromatography-tandem mass spectrometry and application of the technique to comparative pharmacokinetic studies of Radix et Rhei Rhizoma extract alone and Dahuang Fuzi Decoction," Journal of Chromatography B: Analytical Technologies in the Biomedical and Life Sciences, vol. 980, pp. 88-93, 2015.

[29] Y. Jin, C. Qu, Y. Tang et al., "Herb pairs containing Angelicae Sinensis Radix (Danggui): a review of bio-active constituents and compatibility effects," Journal of Ethnopharmacology, vol. 181, pp. 158-171, 2016.

[30] A. K. Drew, A. Bensoussan, I. M. Whyte, A. H. Dawson, X. Zhu, and S. P. S. Myers, "Chinese herbal medicine toxicology database: monograph on Radix Sophorae Flavescentis, 'Ku Shen,' Journal of Toxicology: Clinical Toxicology, vol. 40, no. 2, pp. 173-176, 2002.

[31] G. P. Zhang, S. H. Xie, X. G. Zhu et al., "Experimental study on effects of radix glycyrrhizae on toxicity and efficacy of radix aconiti carmichaeli in compatibility," Chinese Journal of Information on Traditional Chinese Medicine, no. 6, pp. 31-34, 2012.

[32] J. Y. Lee, J.-H. Lee, J. H. Park et al., "Liquiritigenin, a licorice flavonoid, helps mice resist disseminated candidiasis due to Candida albicans by Thl immune response, whereas liquiritin, its glycoside form, does not," International Immunopharmacology, vol. 9, no. 5, pp. 632-638, 2009.

[33] Y.-W. Shin, E.-A. Bae, B. Lee et al., "In vitro and in vivo antiallergic effects of Glycyrrhiza glabra and its components," Planta Medica, vol. 73, no. 3, pp. 257-261, 2007.

[34] S. C. Kim, S. H. Byun, C. H. Yang, C. Y. Kim, J. W. Kim, and S. G. Kim, "Cytoprotective effects of Glycyrrhizae radix extract and its active component liquiritigenin against cadmiuminduced toxicity (effects on bad translocation and cytochrome c-mediated PARP cleavage)," Toxicology, vol. 197, no. 3, pp. 239251, 2004.

[35] L. Shi, X. Tang, X. Dang et al., "Investigating herb-herb interactions: the potential attenuated toxicity mechanism of the 
combined use of Glycyrrhizae radix et rhizoma (Gancao) and Sophorae flavescentis radix (Kushen)," Journal of Ethnopharmacology, vol. 165, pp. 243-250, 2015.

[36] X. Wang, X. Huang, N. Wang et al., "Toxicity of the water extraction of radix sophorae flavescentis formulated with radix glycyrrhizae on mice," Chinese Archives of Traditional Chinese Medicine, vol. 33, no. 7, pp. 1653-1655, 2015. 


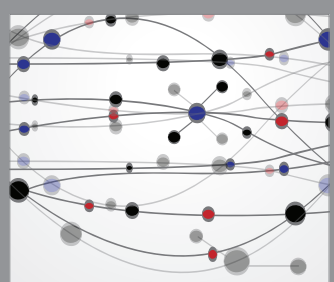

The Scientific World Journal
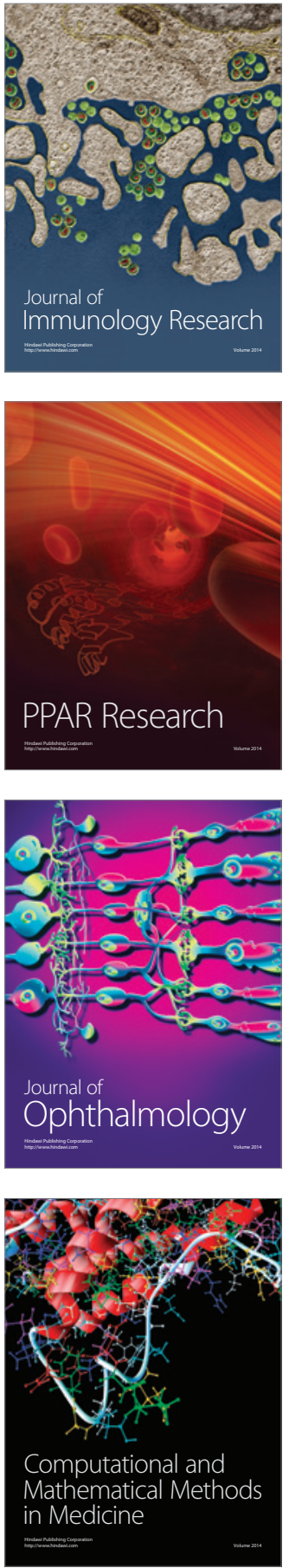

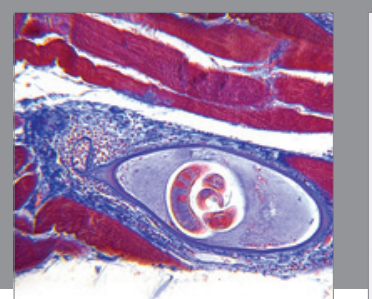

Gastroenterology Research and Practice

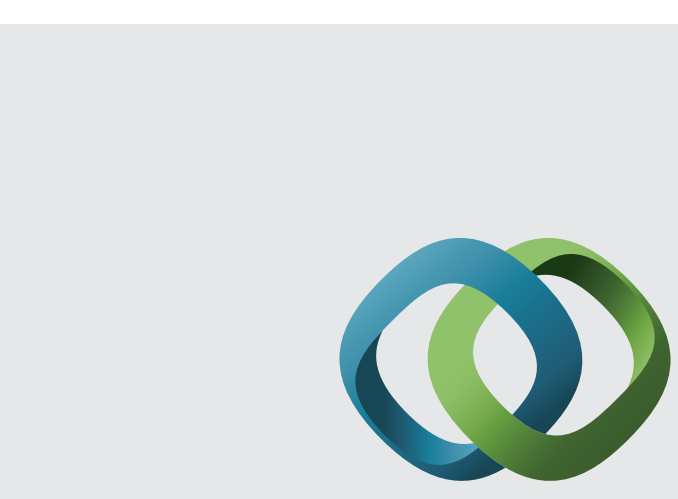

\section{Hindawi}

Submit your manuscripts at

http://www.hindawi.com
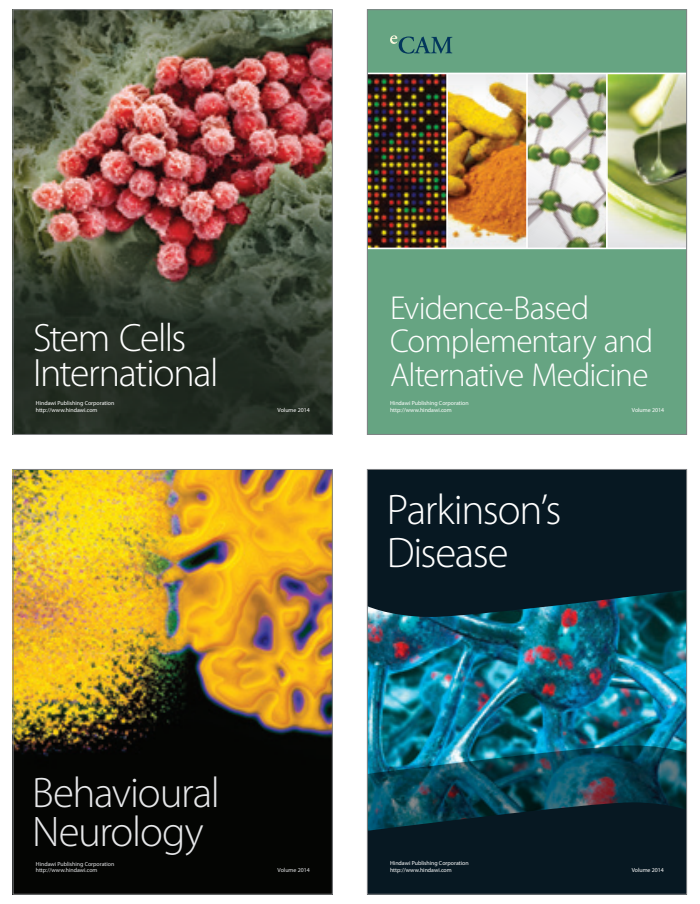
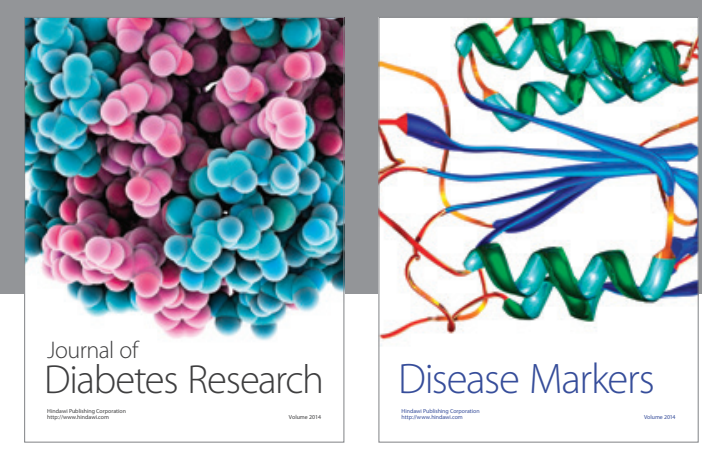

Disease Markers
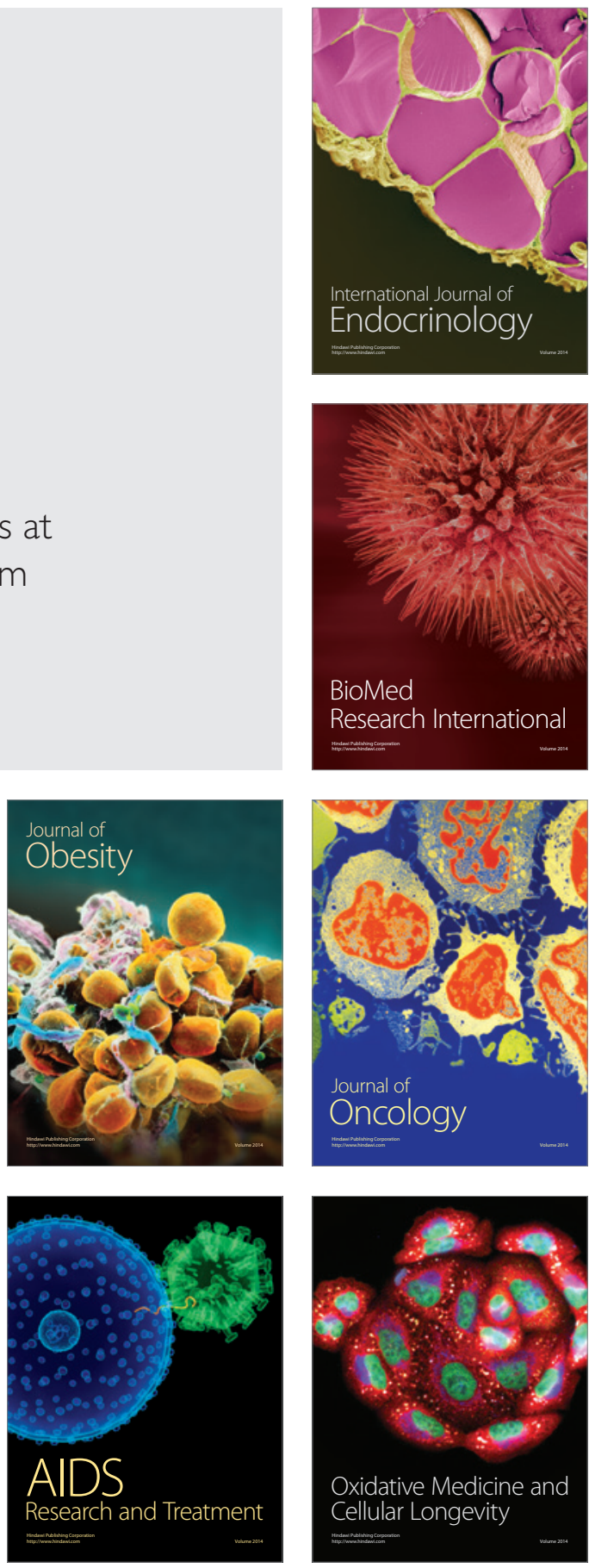